\section{RESTRIÇÕES AO ACESSO INFORMACIONAL: a neutralidade de rede e a prática do zero-rating na governança da Internet ${ }^{1}$}

\author{
Hermann Bergmann Garcia e Silva* \\ Rodrigo Moreno Marques**
}

RESUMO

\begin{abstract}
Com a expansão das ofertas de gratuidade no tráfego de Internet para acesso a determinadas aplicações ou conteúdos, prática denominada de zero-rating, surgem novos desafios para a governança da Internet, notadamente no que se refere à garantia do tratamento isonômico e não discriminatório do fluxo de informações na web. Nesse contexto, o presente trabalho analisa a experiência internacional de normatização do princípio da neutralidade de rede e da prática do zero-rating, a partir dos marcos regulatórios do Canadá, Chile, Holanda, Índia e União Europeia. Para tanto, foi desenvolvida uma pesquisa descritiva de natureza qualitativa, fundamentada em análises bibliográfica e documental. Os resultados apontam, por um lado, para uma tendência de se regulamentar a proibição de práticas na Internet que tenham como objetivo bloquear, priorizar, degradar ou restringir intencionalmente alguns tipos de conteúdo. Por outro lado, verifica-se a ausência de uniformidade no que se refere ao disciplinamento da prática do zero-rating. Nesses termos, a investigação realizada busca contribuir com a discussão acadêmica acerca da regulação de práticas que limitam o acesso informacional dos usuários da Internet.
\end{abstract}

Palavras-chave: Internet - Governança. Internet - Regulação. Rede Neutralidade. Zero-rating.
* Mestre em Sistemas de Informação e Gestão do Conhecimento pela Universidade FUMEC, Brasil. Membro do Grupo de Pesquisa Informação e Conhecimento na Sociedade Contemporânea da Universidade FUMEC, Brasil.

E-mail: tonelero04@hotmail.com.

** Doutor em Ciencia da Informação pela Universidade Federal da Paraíba, Brasil. Professor e pesquisador do Programa de Pós-Graduação em Sistemas de Informação e Gestão do Conhecimento da Universidade FUMEC, Brasil. Coordenador do Grupo de Pesquisa Informação e Conhecimento na Sociedade Contemporânea.

E-mail: rodrigo.marques@fumec.br.

\section{INTRODUÇÃO}

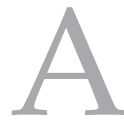

evolução da convergência entre as telecomunicações e as tecnologias da informação e comunicação permitiu que a Internet se tornasse uma plataforma

\footnotetext{
O artigo resulta de mestrado no Programa de Pós-Graduação em Sistemas de Informação e Gestão do Conhecimento da Universidade FUMEC. A pesquisa contou com financiamento da Fundação de Amparo à Pesquisa de Minas Gerais (APQ-02058-17), da Fundação Nacional de Desenvolvimento do Ensino Superior Particular (PROPIC 20I7/2018) e da Universidade FUMEC.
}

transnacional de compartilhamento de informações e conhecimento. $\mathrm{O}$ acesso aberto e não discriminatório, que marcou a concepção original da Internet, também redefiniu a interação entre pessoas, empresas e governos, o que trouxe a perspectiva de florescimento de ambientes inovadores, competitivos e democráticos. O desenvolvimento desse círculo virtuoso foi alicerçado na simplicidade da arquitetura original da Internet (end-to-end), que limitava a atuação de intermediários na rede mundial e conferia maior autonomia ao usuário 
para decidir, de acordo com o seu interesse, quais conteúdos e aplicações seriam acessados ou disponibilizados online (BRAMAN, 2011).

Não obstante, novas tecnologias conferiram aos detentores da infraestrutura da Internet, especialmente empresas provedoras de acesso, uma habilidade sem precedentes de controlar os datagramas que trafegam pela rede mundial, o que influencia a liberdade de escolha dos usuários da web, bem como o conteúdo que nela circula ou deixa de circular. Essas novas tecnologias permitiram a adoção de práticas discriminatórias, por parte dessas empresas, que trazem sérios impactos nas potencialidades da Internet, como o bloqueio de acesso a aplicações, conteúdos ou serviços (blocking), a degradação ou o retardo intencional do tráfego (throttling), a filtragem de conteúdos (filtering) e o tratamento diferenciado a determinadas aplicações e conteúdos.

É nesse contexto que surge o debate em torno da neutralidade de rede. Segundo o princípio da neutralidade de rede os pacotes de dados devem fluir livremente pela Internet, independentemente de seu conteúdo, desde que este seja legal (WU, 2003). Portanto, a racionalidade da neutralidade de rede está consubstanciada na preservação do caráter aberto e descentralizado da Internet, com vistas a empoderar os usuários finais e a preservar os seus direitos e garantias fundamentais (BELLI, 2016).

No centro dessa discussão, insere-se a prática do zero-rating, que se caracteriza como uma modalidade de discriminação por preço que diferencia as condições de usufruto da rede. Por meio do zero-rating, os usuários da Internet não são cobrados ao acessar determinados conteúdos ou aplicações, que são selecionados pelos provedores de acesso em função das suas estratégias de mercado e modelos de negócio.

Considerando a expansão das ofertas de zero-rating em todo mundo e as consequências socioeconômicas dessa tendência em curso (GARCIA E SILVA, 2017), o objetivo do presente artigo é apresentar e discutir as experiências internacionais de normatização da neutralidade de rede e de disciplinamento da prática do zerorating, a partir de uma perspectiva comparada.

Nesse sentido, o artigo apresenta resultados de uma pesquisa de caráter descritivo, com abordagem qualitativa. Os dados compilados ao longo da investigação foram obtidos por meio da conjunção de técnicas de pesquisa bibliográfica e documental, que utilizou como corpus documentos governamentais e informações disponíveis em sítios de Governo e de agências reguladoras. Compõem esse acervo da pesquisa documentos governamentais do Canadá, Chile, Holanda, Îndia e União Europeia, que foram selecionados por já possuírem marcos regulatórios estabelecidos ou por se encontrarem em processo de aprovação e implementação de instrumentos normativos sobre a temática.

A análise realizada revela que há, atualmente, uma tendência de se regulamentar a proibição de práticas discriminatórias na rede mundial que tenham como objetivo bloquear, priorizar, degradar ou restringir intencionalmente o tráfego de dados, que seriam excepcionalizadas em caso de necessidades de natureza técnica, como a de minimizar os efeitos de congestionamento na rede. Todavia, a situação não é a mesma quando a análise se desloca para a aplicação da neutralidade de rede em face das modalidades de discriminação por preço. Nesse universo há uma evidente ausência de uniformidade no que se refere ao disciplinamento de práticas como a do zero-rating.

O texto está estruturado em quatro seções. Após a introdução, a segunda seção faz uma abordagem conceitual do princípio da neutralidade de rede e da prática do zero-rating. A terceira seção discute os marcos regulatórios internacionais que lidam com a neutralidade de rede e com o zero-rating. Na última seção, são tecidas algumas considerações finais sobre $\mathrm{o}$ resultado da pesquisa.

\section{NEUTRALIDADE DE REDE E ZERO- RATING: aspectos conceituais}

A neutralidade de rede reacende o debate sobre as obrigações de empresas que prestam serviços de infraestrutura, que são essenciais para a economia, e como se configura a relação dessas empresas com o interesse público. No século XIX, as ferrovias e os meios de navegação eram o foco da discussão; no século XX, foram a telefonia e a energia elétrica; no século XXI, a Internet assumiu o papel central desse debate (LEE; WU, 2009). 
A persecução conceitual da neutralidade de rede não é uma tarefa trivial, tendo em vista a abrangência dos múltiplos interesses envolvidos na arena da Internet, muitas vezes conflitantes, que tangenciam aspectos de natureza técnica, econômica e de direitos e garantias fundamentais (BRAMAN, 2006). Dependendo da perspectiva de análise, têmse interpretações distintas, o que torna a sua definição um importante objeto de pesquisa para a compreensão da dinâmica contemporânea das relações socioeconômicas na rede mundial de computadores.

A discussão sobre a neutralidade de rede iniciou-se nos Estados Unidos, no início da década de 2000, e estava centrada na questão de se definir em até que extensão os detentores da infraestrutura de telecomunicações podem interferir no tráfego da rede mundial ou mesmo estabelecer modelos de remuneração sem interferir na competição do mercado, na livre circulação da informação na Internet e na liberdade de escolha dos usuários.

De acordo com o princípio da neutralidade de rede, a Internet, como uma plataforma de compartilhamento de informações, deve transmitir os datagramas de maneira isonômica, independentemente da sua origem, destino, conteúdo, aplicação ou serviço, desde que a rede possua capacidade disponível para transportálos. O referido princípio busca preservar os fundamentos que tornaram a Internet um instrumento de incentivo à inovação, de diminuição das barreiras de comunicação e de empoderamento do usuário final.

Ramos (2014) identifica três princípios constitutivos da ideia de neutralidade de rede, que podem ser sistematizados na obrigação imposta aos operadores da rede de não bloquear ou reduzir a velocidade de acesso de usuários a determinados conteúdos ou aplicações; na vedação de cobrança de tarifas diferenciadas para acesso a determinados conteúdos ou aplicações; e na obrigação de se manter práticas transparentes e razoáveis de gerenciamento de tráfego.

Também é destacado por esse autor o fato de os provedores de acesso à Internet controlarem a última milha (last mile) da infraestrutura de telecomunicações, ou seja, o segmento da rede que faz a conexão com os usuários, o que lhes confere a capacidade técnica e operacional de filtrar ou controlar o fluxo de comunicação na rede mundial de computadores (RAMOS, 2015). A circunstância de os provedores de acesso à Internet serem os detentores da última milha da infraestrutura de telecomunicações possibilita que eles se tornem verdadeiros guardiões (gatekeepers) da rota de conectividade para os usuários finais, da qual são dependentes os provedores de aplicações e de conteúdo (PRAKASH, 2015). Van Schewick (2016) entende que, ao atuar como guardiões, os provedores de acesso à Internet podem utilizar a sua posição privilegiada para decidir quem serão os ganhadores e perdedores da competição no mercado, favorecendo algumas aplicações em detrimento de outras.

Os argumentos favoráveis à neutralidade de rede se fundamentam no livre acesso à circulação da informação e serviços na Internet, que permitem o exercício de direitos fundamentais dos cidadãos, além de uma rede neutra viabilizar um ambiente inovador que privilegia a competição. Por outro lado, o desestímulo ao investimento na infraestrutura de rede, a necessidade de gerenciamento do tráfego, do uso eficiente de um recurso escasso e o atendimento a novos serviços são elencados como os principais fundamentos contrários à neutralidade de rede.

Já a ausência do princípio da neutralidade de rede possibilita a prática de discriminação do tráfego pelas operadoras de telecomunicações, por meio de acordos comerciais com os provedores de conteúdo, o que impacta a livre circulação da informação e do conhecimento e coloca em risco a liberdade de escolha do usuário (MARQUES; KERR PINHEIRO, 2014).

No contexto atual da neutralidade de rede, um tema que tem adquirido destaque é a prática do zero-rating pelos provedores de acesso à Internet, que consiste na aplicação de gratuidade no tráfego de dados associado a uma aplicação, a um serviço ou a um conjunto de aplicações, cujo consumo de dados não é contabilizado na franquia mensal contratada ou na conexão à Internet realizada pelos usuários. A prática é um modelo diferenciado de precificação em que o custo de utilização da infraestrutura de telecomunicações é absorvido pelo provedor de acesso ou pelo provedor de conteúdo.

Para a Agência Nacional de Telecomunicações (Anatel), o conceito de zerorating é sistematizado nos seguintes termos: 
O zero-rating é uma prática realizada por prestadoras de serviços de telecomunicações que consiste em aplicar um preço zero para o tráfego de dados móveis associado a uma aplicação ou classe de aplicações em particular, implicando na não contagem desse tráfego para efeitos de uma franquia de dados (data cap) eventualmente aplicada ao acesso à internet contratado (ANATEL, 2016, p. 2).

A prática do zero-rating pode ser configurada em diversos arranjos comerciais, em função da relação do provedor de acesso à Internet e do provedor de conteúdo. Eisenach (2015) destaca que os dois tipos mais comuns da prática são aqueles em que o acesso gratuito é estabelecido pela própria operadora de telecomunicações (carrier initiated), com o objetivo de atrair potenciais novos consumidores para aumentar a sua participação no mercado, e o denominado acesso patrocinado (sponsored data), em que o provedor de conteúdo subsidia o custo de acesso dos usuários para disponibilizar o seu conteúdo de forma gratuita. Nas duas modalidades, o acesso às aplicações ou ao conteúdo não onera financeiramente o usuário final, nem mesmo após atingir o limite de dados contratados com a operadora, situação em que as demais aplicações têm a velocidade de tráfego reduzida ou são bloqueadas.

Na visão de Ramos (2015), o zero-rating pode trazer pelo menos três consequências negativas para a livre circulação da informação na Internet, a saber: a possibilidade de governos utilizarem essa prática para controlar o fluxo de informações na Internet, influenciando o consumo de apenas determinados conteúdos; a constituição de barreiras sociais, com uma potencial divisão entre uma "Internet dos ricos", com amplo acesso informacional, e outra "Internet dos pobres", em que a experiência da Internet se limitaria a determinados conteúdos; e a criação do chamado efeito walled garden, em que os usuários teriam pouco incentivo para explorar livremente conteúdos na Internet, limitando-se a utilizar as aplicações ou serviços que não tivessem impacto na franquia de dados contratada. Destaca-se que o efeito walled garden ocorre quando o usuário tem uma experiência da Internet muito restrita ou controlada por filtros de conteúdos, que impede o usufruto da rede mundial de computadores como um ambiente livre, colaborativo e democrático.

Belli (2016) utiliza a referência do Minitel a fim de analisar as consequências da prática do zero-rating para a neutralidade de rede e para a natureza da Internet, notadamente no que se refere às suas características de uma rede aberta, descentralizada e fundada na interoperabilidade. O sistema Minitel foi muito popular na França na década de 1980 e se constituiu em uma rede fechada de comunicação em que a empresa estatal detentora da infraestrutura decidia quais serviços estariam disponíveis para os usuários finais. Ao final de 1985, existiam 1.3 milhão de terminais em utilização no país (HART, 1988).

Esse autor cunhou o termo "minitelização" (minitelisation) para definir o fenômeno de evolução da Internet de uma rede de propósito genérico para uma rede com um propósito específico, caracterizada por um controle centralizado em que os usuários deixariam de participar ativamente da rede e se tornariam consumidores passivos de conteúdos e aplicações pré-selecionadas pelos provedores de acesso.

Como uma rede de propósito genérico, a forma como a Internet é utilizada não é predefinida pela empresa de telecomunicações. Pelo contrário, a sua utilização é estabelecida de maneira autônoma pelos usuários finais e provedores de conteúdo. Por conseguinte, a concepção original da Internet empoderou os usuários ao conferir-lhes não apenas a habilidade de acessar a rede mundial de computadores, mas também de criar e compartilhar informações, aplicações e serviços, sem a necessidade de permissão dos detentores da infraestrutura.

Segundo Belli (2016), a minitelização da Internet pode resultar não apenas de práticas de gerenciamento do tráfego (bloqueio, priorização e degradação) como também de modalidades de discriminação por preço, como o zero-rating, já que o objetivo conjugado de se estabelecerem limites para o consumo de dados com o acesso patrocinado a determinadas aplicações serviria para influenciar a escolha dos usuários, o que causaria uma fragmentação da Internet em domínios privados que seriam definidos unilateralmente pelos operadores da rede. 


\section{A REGULAÇÃO DA NEUTRALIDADE DE REDE E DO ZERO-RATING NO CENÁRIO INTERNACIONAL}

A discussão sobre a normatização do princípio da neutralidade de rede e o disciplinamento da prática do zero-rating compõem uma das controvérsias mais atuais na arena da Governança da Internet e integra a agenda regulatória de diversos países, dentre os quais se destacam: Canadá, Chile, Holanda e Índia, além da União Europeia.

\section{I Canadá}

Os primeiros registros de disputa envolvendo a neutralidade de rede no Canadá ocorreram em 2005, quando a Telus, segunda maior empresa de telecomunicações do país, decidiu bloquear o sítio eletrônico Voices for Change, do sindicato de trabalhadores do setor, pelo fato de estar em processo de litigância trabalhista com a entidade. No mesmo ano, o provedor de acesso à Internet via cabo Shaw Communications anunciou um modelo de negócio que criava um serviço de voz sobre o protocolo IP (Voice over IP - VoIP) por meio do qual, mediante pagamento de assinatura mensal, a transmissão de voz do usuário era priorizada em relação ao tráfego de dados e vídeo que circulava pela rede da operadora (GEIST, 2008).

Em 2008, a Associação Canadense de Provedores de Internet apresentou uma reclamação administrativa à Canadian RadioTelevision and Telecommunications Commission - CRTC, alegando que a Bell Canada utilizava práticas de restrição do tráfego de rede (traffic shaping) para degradar a velocidade de transmissão dos pacotes associados às aplicações de transferência de arquivos nos serviços de banda larga fixa comercializados no atacado. A Bell Canada reconheceu a prática, sustentando que a medida era necessária nos horários de maior volume de tráfego para evitar o congestionamento da rede. Ao analisar a questão, nos termos da Telecom Decision 2008-108 (CRTC, 2008), a autoridade reguladora canadense concluiu que a medida utilizada pela Bell Canada para administrar a rede não violava as regras estabelecidas na legislação canadense.
Em 2009, reconhecendo a importância da matéria, a CRTC aprovou uma política de telecomunicações para disciplinar o uso de práticas de gerenciamento de tráfego de Internet (Internet Traffic Management Policy - ITMP), consubstanciada na Telecom Regulatory Policy 2009-657 (CRTC, 2009), que regulamenta o disposto no Telecommunications Act:

27(2) Nenhuma operadora canadense
deve, em relação à prestação de serviços
de telecomunicações ou à cobrança
pelos serviços prestados, injustamente
discriminar ou conceder uma preferência
excessiva ou não razoável para qualquer
pessoa, incluindo a si mesma, ou sujeitar
qualquer pessoa a uma desvantagem
excessiva ou não razoável.
[...]
36 Exceto quando a Comissão aprovar
de forma diferente, uma operadora
canadense não deve controlar o
conteúdo ou influenciar o significado
ou o propósito das telecomunicações
transportadas por ela para o público
(CANADA, I993, p. 18 e 23 , tradução
nossa).

No que se refere especificamente ao zero-rating, a CRTC estabeleceu, em 2015, um importante precedente por meio da decisão Brocasting and Telecom Decision (CRTC, 2015), que considerou a prática das empresas Videotron e Bell Mobility, de discriminar aplicativos de streaming de vídeo em favor de suas respectivas plataformas, como uma violação à estrutura normativa canadense, notadamente do disposto na Seção 27(2) do Telecommunications Act de 1993.

Por meio da Telecom Notice of Consultation 2016-192 (CRTC, 2016), a autoridade reguladora do Canadá realizou uma audiência pública com o objetivo de discutir a formulação de uma política regulatória para disciplinar as práticas de discriminação de preços pelos provedores de acesso à Internet e avaliar se seria adequado estabelecer um disciplinamento ex-ante para o zero-rating, ao invés de se ter uma abordagem caso a caso para a questão.

Em 2017, com a aprovação da Telecom Regulatory Policy 2017-104 (CRTC, 2017), foram estabelecidos os critérios para avaliação de práticas de discriminação por preço, que se referem tanto ao zero-rating quanto a descontos no mercado de varejo para o tráfego de dados na Internet. A CRTC instituiu uma regulação ex-post, 
ou seja, os critérios de exclusividade da oferta, de impacto na inovação e abertura da Internet, de compensação financeira entre os provedores de acesso e provedores de conteúdo e de tratamento agnóstico no tráfego de dados deverão ser analisados a partir de cada caso concreto pela autoridade reguladora do Canadá.

\subsection{Chile}

Silva (2013) destaca que o regramento normativo da neutralidade de rede no Chile foi o resultado de uma sucessão de fatos, em que os principais provedores de acesso à Internet do país foram questionados pela falta de qualidade na prestação do serviço e pela ausência de transparência nas suas operações comerciais. Esse autor ressalta que, em 2003, já se registravam no país práticas de bloqueio e degradação de tráfego pela Telefônica, que controlava grande parte dos serviços de telefonia fixa e das conexões à rede mundial no país, em detrimento de empresas concorrentes que transmitiam voz digitalizada sobre a Internet (VoIP).

O processo de discussão legislativa do referido marco regulatório se iniciou em 2007 e contou com uma participação multissetorial, incluindo tanto usuários e organizações não governamentais como também associações representativas dos provedores de acesso à Internet e das empresas de telecomunicações, além de incluir a autoridade reguladora do Chile, a Subsecretaria de Telecomunicações - Subtel, órgão do Ministério de Transporte e Telecomunicações, e o próprio Parlamento chileno.

Em 2010, o Chile se tornou o primeiro país a promulgar uma lei para regular a Internet, que instituiu oficialmente o princípio da neutralidade de rede. A Lei $\mathrm{n}^{\circ} 20.453$, publicada em 26 de agosto, acrescentou os artigos 24h, 24i, 24j à Lei Geral de Telecomunicações do país (Lei $n^{\circ}$ 18.168/82).

O referido marco regulatório estabeleceu que os provedores de acesso à Internet não podem arbitrariamente bloquear, interferir, discriminar, impedir ou restringir o direito de qualquer usuário de utilizar, enviar, receber ou oferecer qualquer conteúdo, aplicação ou serviço através da Internet, nos seguintes termos:

\begin{abstract}
Artigo $24 \mathrm{H}$ -
As concessionárias de serviço público de telecomunicações que prestam serviço aos provedores de acesso à Internet, bem como esses últimos, entendidos como toda pessoa natural ou jurídica que prestem serviços comerciais de conexão entre os usuários ou suas redes e a Internet:

a) Não podem arbitrariamente bloquear, interferir, discriminar, impedir nem restringir o direito de qualquer usuário da Internet de utilizar, enviar, receber ou oferecer qualquer conteúdo, aplicação ou serviço legal através da Internet, assim como qualquer outro tipo de atividade ou uso legal realizado através da rede. Nesse sentido, deverão oferecer a cada usuário um serviço de acesso à Internet ou de conexão ao provedor de acesso à Internet, de acordo com o caso, que não distinga arbitrariamente conteúdos, aplicações ou serviços, seja com base na origem ou propriedade dos mesmos, levando em consideração as diferentes configurações de conexão à Internet segundo $\mathrm{o}$ contrato vigente com os usuários (CHILE, 2010, p. I, tradução nossa).
\end{abstract}

Em 15 de dezembro de 2010, foi aprovado o Decreto $n^{\circ} 368$, que regulamentou as características e condições da neutralidade de rede, sendo definidas no artigo $8^{\circ}$ as práticas consideradas incompatíveis com a Lei $n^{\circ}$ 20.453/2010:

- Toda ação que tenha como objetivo bloquear, interferir, impedir, restringir ou de qualquer forma obstaculizar o uso, o envio, a recepção ou a oferta de qualquer conteúdo, aplicação ou serviço através da Internet, assim como práticas de gestão de tráfego e administração da rede que afetem os níveis de serviço contratados pelos usuários.

- Toda ação que, de forma arbitrária, seja direcionada a priorizar ou discriminar provedores de conteúdos, aplicações ou usuários, particularmente aqueles da mesma natureza.

- $\quad$ Toda ação que impeça ou restrinja o direito dos usuários de acessar a informação fidedigna e atualizada referente às características dos serviços de acesso à Internet ofertados ou contratados (CHILE, 2010). 
Em janeiro de 2013, entidades não governamentais que promovem a neutralidade de rede no Chile protocolaram requerimentos na Subtel questionando a prática do zero-rating pela empresa prestadora de telefonia móvel Virgin Mobile, que estaria em desacordo com os princípios de neutralidade de rede aprovados no país. A argumentação dos requerentes alegava que a referida prática discriminava, de maneira arbitrária, um provedor de conteúdo em relação aos demais.

Instada a se pronunciar sobre os planos de telefonia móvel que ofertavam acesso gratuito a aplicações ou serviços específicos na Internet, a Subtel fixou o entendimento de que essas ofertas comerciais têm um caráter eminentemente discriminatório ao beneficiar uma aplicação específica em detrimento das demais, sendo caracterizadas como uma violação ao princípio da neutralidade de rede (SUBTEL, 2014).

\subsection{Holanda}

A Holanda é reconhecida como o segundo país no mundo e o primeiro da União Europeia a garantir a neutralidade de rede através de uma lei. Em 2012, foi aprovada pelo Senado holandês uma alteração do Telecomunications Act de 1998 (Telecommunicatiewet), que incluiu o artigo 7.4a na redação original.

A motivação para a emenda foi alicerçada na intenção de empresas de telefonia móvel de cobrar preços diferenciados para o acesso a aplicativos como o WhatsApp e Skype, que concorriam com os seus próprios serviços de telefonia tradicional. Outro fato que impulsionou a iniciativa foi a utilização pelas operadoras de telefonia móvel de técnicas de inspeção profunda de pacotes (Deep Packet Inspection DPI) para distinguir a natureza dos pacotes transmitidos na Internet, com vistas a priorizar o conteúdo trafegado na rede (VAN EIJK, 2014).

Publicada em 4 de junho de 2012, a referida alteração passou a vigorar a partir de $1^{\circ}$ de janeiro de 2013, sendo estabelecidas diretrizes que vedam práticas discriminatórias dos provedores de comunicações eletrônicas no país, a saber:
Artigo 7.4a

I. Provedores de redes públicas de comunicações eletrônicas, através das quais os serviços de acesso à Internet são prestados, e provedores de acesso à Internet não devem obstruir ou retardar aplicações ou serviços na Internet, a menos e na extensão que a medida em questão com a qual aplicações ou serviços estão sendo obstruídas ou retardadas seja necessária:

a. para minimizar os efeitos de congestionamento, através do qual tipos iguais de tráfego devem ser tratados de maneira isonômica;

b. para preservar a integridade e segurança da rede e do serviço do provedor em questão ou do usuário final do terminal;

c. para restringir a transmissão de um usuário final de comunicações não solicitadas sob as disposições do Artigo II.7(I), desde que o usuário final tenha dado o seu consentimento prévio para que isso seja feito, ou

d. para implementar uma disposição legal ou decisão judicial.

[...]

3. Provedores de serviço de acesso à Internet não devem fazer suas cobranças pelo serviço de acesso à Internet em função dos serviços e aplicações que são oferecidos ou usados por meio do referido serviço (HOLANDA, 20I2, p. 44, tradução nossa).

Como se verifica no parágrafo primeiro do artigo $7.4 \mathrm{a}$, os provedores de acesso à Internet não podem obstruir ou retardar o tráfego da Internet, a não ser em situações excepcionais para: minimizar os efeitos de congestionamento na rede; preservar a segurança e a integridade da rede, do serviço ou do usuário final; restringir comunicações não solicitadas (spam) para o usuário final; para implementar uma disposição legal ou decisão judicial.

Já no parágrafo terceiro, é vedada a vinculação do preço de acesso à Internet a serviços ou aplicações específicas que são ofertadas ou utilizadas pelos usuários finais. Assim, o dispositivo legal proíbe qualquer modalidade de discriminação por preço na Holanda, o que inclui a prática do zero-rating.

A regulamentação das regras dispostas no Telecommunications Act somente foi aprovada em maio de 2015, por meio de um Decreto 
do Ministro de Assuntos Econômicos, que estabeleceu orientações gerais sobre a aplicação da neutralidade de rede na Holanda, com vistas a subsidiar a Autoridade para Consumidores e Mercado - ACM na implementação e fiscalização das regras dispostas no artigo 7.4a do Telecomunications Act (FOUAD, 2015).

Em 2016, o Tribunal de Justiça do Distrito de Roterdã confirmou uma decisão da ACM, que impôs uma multa de €200.000 à operadora Vodafone, por ofertar o aplicativo de vídeo HBO GO aos seus usuários sem descontar o tráfego de dados do plano de serviço contratado. Em sua fundamentação, o Tribunal acompanhou o entendimento da autoridade reguladora de que a prática de zero-rating viola o disposto no artigo 7.4a, parágrafo terceiro, do marco regulatório, já que constitui uma modalidade de discriminação por preço (ACM, 2016a).

Em outra decisão recente, a ACM determinou que a operadora T-Mobile interrompesse a oferta do serviço de streaming de música Data-free Music, lançado comercialmente em 10 de outubro de 2016, tendo em vista a violação das regras de neutralidade de rede no país, caracterizada pela prática do zero-rating, sob pena de aplicação de multa diária de $€ 50.000$ (ACM, 2016b).

\section{4 Índia}

Em 2015, um relatório da comissão formada pelo Departamento de Telecomunicações do Ministério das Comunicações (ÍNDIA, 2015) já sinalizava a possibilidade de ação regulatória pelo governo indiano, ao recomendar a elaboração de princípios fundamentais para a neutralidade de rede no país, de forma que os direitos dos usuários da Internet fossem garantidos, com vistas a evitar que empresas de telecomunicações pudessem, de alguma maneira, restringir a habilidade do usuário de enviar, receber, disponibilizar, usar ou compartilhar qualquer conteúdo de origem legal na rede mundial de computadores. Outra recomendação feita pelo relatório se refere às práticas de gerenciamento de tráfego que deveriam ser utilizadas somente nos casos em que não existissem conflitos com a neutralidade de rede.

Marsden (2016) destaca o pronunciamento à época do Secretário Adjunto do Departamento de Telecomunicações, Sr. V. Umashankar, que também participou como membro efetivo da comissão, indicando a possibilidade de uma regulação ex-ante a partir dos subsídios fornecidos pelo referido relatório, a saber: "Como dissemos no relatório, se for necessário, o governo e a autoridade reguladora podem intervir para restaurar o equilíbrio para garantir que a Internet continue a ser uma plataforma aberta e neutra para a liberdade de expressão e inovação" (DOVAL, 2015, tradução nossa).

Após um intenso período de debates, que durou mais de onze meses, entre as entidades que defendiam a neutralidade de rede, as empresas de telecomunicações e os provedores de conteúdo, especialmente os promotores do Free Basics, a autoridade reguladora da Índia (TRAI) publicou, em 2016, uma norma proibindo a discriminação por preço nos serviços de dados no país, o que inclui a vedação ao zero-rating, nos termos especificados no artigo $3^{\circ}$ :

\section{Proibição de discriminação de tarifas}

(I) Nenhum provedor de serviço deve oferecer ou cobrar tarifas discriminatórias para serviços de dados nos serviços de conteúdo.

Nenhum provedor de serviço deve celebrar qualquer negócio, acordo ou contrato, qualquer que seja o nome atribuído, com qualquer pessoa, natural ou jurídica, que tenha o efeito de discriminar tarifas pelos serviços de dados que são ofertados ou cobrados do consumidor com base no conteúdo:

Fica estabelecido que este regulamento não será aplicável para os serviços de dados em redes eletrônicas de comunicações fechadas, a menos que tais tarifas sejam ofertadas ou cobradas pelo provedor de serviço com a finalidade de evitar a proibição neste regulamento.

(3) A decisão da Autoridade sobre se um provedor de serviços está em descumprimento ao disposto neste regulamento deve ser definitiva e vinculativa (TRAI, 2016, p.2, tradução nossa).

O conceito de serviços de dados na norma em destaque abrange qualquer serviço ofertado ou prestado ao consumidor para acessar ou transmitir conteúdos na Internet, utilizando qualquer equipamento, tecnologia ou meio, incluindo tecnologias com e sem fio. 
Em 2017, a TRAI realizou uma consulta pública para subsidiar a formulação de uma política para a neutralidade de rede no país (TRAI, 2017). Com a iniciativa, a autoridade reguladora da Índia tem a intenção de obter contribuições dos principais atores sociais em quatro tópicos principais, quais sejam: práticas de gerenciamento de tráfego, princípios fundamentais da neutralidade de rede, transparência e abordagem regulatória.

Recentemente, em novembro de 2017, a partir da referida consulta pública, foram apresentados pela TRAI as recomendações para a neutralidade de rede na Índia, entre as quais se destaca:

Capítulo 7 - Sumário das
Recomendações
Os serviços de acesso à Internet
devem ser governados por um
princípio que restrinja qualquer forma
de discriminação ou interferência no
tratamento do conteúdo, incluindo
práticas como o bloqueio, a degradação,
o retardo ou a concessão preferencial de
velocidade ou tratamento de qualquer
conteúdo (TRAI, 2007, p. 44, tradução
nossa).

\subsection{União europeia}

Em 2015, foi aprovada pelo Parlamento Europeu e Conselho da União Europeia a Regulation 2015/2120, com o objetivo de estabelecer normas comuns para preservar o tratamento isonômico e não discriminatório do tráfego na Internet, de forma a proteger os usuários finais e, ao mesmo tempo, garantir a continuidade da Internet como uma plataforma aberta para a inovação, conforme especificado nesse instrumento normativo (UNIÃO EUROPEIA, 2015, p. 310/08).

Em 2016, o Body of European Regulators of Electronic Communications - BEREC colocou em consulta pública uma proposta de regulamentação da Regulation 2015/2120, com vistas a servir de referência para a implementação das regras a serem estabelecidas pelas Autoridades Reguladoras Nacionais dos vinte e oito Estados-Membros da União Europeia (BEREC, 2016a).

Em agosto de 2016, após 481.547 contribuições recebidas dos mais diversos setores da sociedade, as quais representaram uma participação sem precedentes nas consultas públicas já realizadas pela entidade, foi publicado o documento final de orientações gerais que foi denominado de Guidelines on the Implementation by National Regulators of European Net Neutrality Rules (BEREC, 2016b).

A partir da análise das orientações gerais publicadas, verifica-se que a União Europeia não estabeleceu uma proibição absoluta do zerorating, mas especificou os tipos que não seriam compatíveis com as regras de neutralidade de rede, além de definir critérios de referência para que as Autoridades Reguladoras Nacionais pudessem avaliar, ex-post, em cada caso concreto, quais práticas seriam ou não admitidas.

Há que se ressaltar que a Regulation 2015/2012, dispõe que contratos entre provedores de acesso à Internet e usuários finais, em relação às condições técnicas e comerciais para provimento do serviço, como preço, volume de dados e velocidade, não podem restringir a liberdade de escolha de conteúdos e informações que os usuários desejam acessar ou distribuir e nem discriminar aplicações ou serviços:

\section{Artigo $3^{\circ}$}

Garantia de acesso à Internet aberta

[...]

2. Os acordos entre os provedores de serviços de acesso à Internet e os usuários finais sobre as condições comerciais e técnicas e sobre as características dos serviços de acesso à Internet, tais como preço, volume de dados ou velocidade, e quaisquer práticas comerciais utilizadas por provedores de serviços de acesso à Internet, não devem limitar o exercício do direito dos usuários finais previsto no parágrafo primeiro (UNIÃO EUROPEIA, 2015, p. 310/8, tradução nossa).

Já o parágrafo terceiro, do mesmo artigo, prevê que provedores de acesso à Internet devem tratar o tráfego da rede de forma isonômica, sem discriminação, restrição ou interferência, inclusive no que se refere ao conteúdo acessado ou distribuído, nos seguintes termos:

Artigo $3^{\circ}$

Garantia de acesso à Internet aberta [...]

3. Os provedores de serviços de acesso à Internet devem tratar todo o tráfego de maneira isonômica, ao prestarem 
serviços de acesso à Internet, sem discriminação, restrição ou interferência, independentemente do emissor e do receptor, do conteúdo acessado ou distribuído, das aplicações ou serviços utilizados ou prestados, ou do equipamento terminal utilizado (UNIÃO EUROPEIA, 2015, p. 310/8, tradução nossa).

Portanto, nos termos estabelecidos no marco regulatório para a neutralidade de rede, na situação em que todas as aplicações são bloqueadas ou a velocidade de acesso reduzida quando se alcança o limite de dados contratados, o fato de aplicações zero-rated não serem submetidas a esse bloqueio ou redução caracteriza uma violação ao disposto no artigo $3^{\circ}$, parágrafo terceiro, da Regulation 2015/2120.

No mesmo sentido, o BEREC (2016b) estabelece que a atribuição do zero-rating a apenas determinadas aplicações também viola o previsto no artigo $3^{\circ}$, parágrafo segundo, já que a sua prática limita o direito dos usuários ao acesso e à distribuição de conteúdos na Internet. Por outro lado, se o zero-rating for aplicado a toda uma classe de aplicações de mesma natureza, por exemplo, a todas as aplicações de streaming de vídeo, o BEREC (2016b) considera que a prática está em consonância com o princípio da neutralidade de rede, já que não se evidenciaria uma discriminação específica de determinada aplicação.

Na União Europeia, a análise de outras variedades de zero-rating deverá ser realizada a partir da apreciação dos casos concretos pelas autoridades reguladoras nacionais dos EstadosMembros. Para tanto, foram estabelecidos critérios de referência que deverão ser utilizados para verificar se a prática é ou não permitida sob a égide da Regulation 2015/2120. Esses critérios incluem: avaliar se a prática é incompatível com os objetivos da norma de salvaguardar o tratamento isonômico e não discriminatório do tráfego e garantir o funcionamento da Internet como uma plataforma aberta para a inovação; avaliar o poder de mercado das entidades envolvidas; verificar se a prática impõe uma redução na oferta de aplicações disponíveis e se há uma redução na possibilidade de escolha dos usuários; verificar se a prática impõe restrições ao conteúdo que os provedores de aplicações podem veicular e se eles serão desestimulados a entrar no mercado; identificar a abrangência da prática e as alternativas que os usuários teriam de acesso a outros planos de serviços (BEREC, 2016b).

\section{CONSIDERAÇÕES FINAIS}

A análise de marcos regulatórios internacionais demonstra que há, atualmente, uma tendência de se regulamentar a proibição de práticas discriminatórias na rede mundial que tenham como objetivo bloquear, priorizar, degradar ou restringir intencionalmente o tráfego de dados, que seriam excepcionalizadas por situações de natureza técnica, como a de minimizar os efeitos de congestionamento na rede.

Não obstante, a situação não é a mesma quando a análise se desloca para a aplicação da neutralidade de rede em face das modalidades de discriminação por preço. Nesse universo há uma evidente ausência de uniformidade no que se refere ao disciplinamento de práticas como a do zero-rating. Enquanto países como a Índia, a Holanda e o Chile buscam a intervenção antecipatória (regulação ex-ante), de maneira a consolidar a proibição do zero-rating, outros como o Canadá e a própria União Europeia relegam a análise dessa prática para o caso concreto (regulação ex-post), com vistas a avaliar quais condutas seriam ou não admitidas.

Essa situação evidencia as tensões entre os atores que compõem a infraestrutura da Internet que, pautados por interesses próprios, buscam influenciar a abrangência e a implementação das salvaguardas do princípio da neutralidade de rede, o que resulta na proliferação de modelos de negócio que restringem a circulação de informação na Internet.

Nesse contexto, a governança da Internet se torna mediadora desse amplo espectro de interesses, que coloca em risco o caráter aberto, democrático, descentralizado e isonômico que marcou as primeiras décadas de funcionamento da rede mundial de computadores, o que torna imperioso o desenvolvimento de uma estrutura normativa que alcance um equilíbrio entre direitos e responsabilidades dos principais atores do ambiente digital, de maneira a não restringir a liberdade de escolha de conteúdos como parte indissociável do processo de produção e disseminação de informação e conhecimento. 
Assim, percebe-se que o futuro da Internet está em jogo e, desse embate que está em curso, poderá emergir uma rede mundial cada vez mais distante dos seus pressupostos originais de uma rede aberta e decentralizada, que possibilitou o surgimento de um espaço de ampla participação democrática. A expansão da prática do zero-rating poderá configurar um cenário de fragmentação da Internet em domínios privados de conteúdos e aplicações patrocinados, que seriam definidos unilateralmente pelos provedores de acesso, restringindo a liberdade de escolha e o acesso informacional dos usuários.

Artigo recebido em 16/02/20I8 e aceito para publicação em 05/04/20I8

\section{INFORMATION ACCESS RESTRICTIONS: net neutrality and zero-rating practice in Internet governance}

ABSTRACT With the expansion of Internet bundles that are free of charge for certain applications or content, practice known as zero-rating, new challenges arise for the governance of Internet, especially with regard to equal and non-discriminatory treatment of information flow on the web. In this context, the present paper analyzes the international experience in regulating the net neutrality principle and the zero-rating practice from the regulatory frameworks of Canada, Chile, Netherlands, India and European Union. Therefore, a descriptive research of qualitative nature was developed, based on bibliographic and documentary analyses. The results indicate, on the one hand, a tendency to regulate the prohibition of Internet practices that aim to block, prioritize, degrade or intentionally restrict some types of content. On the other hand, a lack of uniformity is verified in relation to zerorating regulation. In these terms, the research seeks to contribute to the academic discussion about the regulation of practices that limit the information access of Internet users.

Keywords: Internet governance. Internet Regulation. Net neutrality. Zero-rating.

\section{REFERÊNCIAS}

ACM. Rulling of the District Court of Rotterdam. 2016a. Disponível em: <https://www.acm.nl/en/publications / publication/15459/District-Court-of-Rotterdamrules-in-net-neutrality-case/>. Acesso em: 9 mar. 2017.

ACM. ACM forces T-Mobile to stop its Datafree Music service. 2016b. Disponível em: <https://www.acm.nl/en/publications / publication/16810/ ACM-forces-T-Mobile-tostop-its-Data-free-Music-service/>. Acesso em: 09 mar. 2017.

ANATEL. Informe $n^{\circ}$ 4/2016/SEI/SCP. Brasília, 2016.
BELLI, Luca. Net neutrality, zero rating and the Minitelisation of the internet. Journal of Cyber Policy, p. 1-27, 2016.

BEREC. Public Consultation on draft Berec Guidelines on implementation by National Regulators of European net neutrality rules. Riga, 2016a. Disponível em: <http://berec. europa.eu/eng/document_register/subject_ matter/berec/public_consultations/6075-draftberec-guidelines-on-implementation-by-nationalregulators-of-european-net-neutrality-rules>. Acesso em: 20 jun. 2016.

BEREC. Guidelines on the Implementations by National Regulators of European Net Neutrality Rules. Riga, 2016b. Disponível em: <http://berec. europa.eu/eng/document_register/subject_matter/ berec/regulatory_best_practices/guidelines/6160- 
berec-guidelines-on-the-implementation-bynational-regulators-of-european-net-neutralityrules>. Acesso em: 20 jun. 2016.

BRAMAN, Sandra. Change of State Information, Policy and Power. Cambridge, MA: MIT Press, 2006.

Internet policy. In: COSALVO, Mia; ESS, Charles (Ed.). The handbook of internet studies. Oxford: John Wiley \& Sons, 2011. p. 137-167.

CANADA. Telecommunications Act, S.C., 1993, C. 38. Ottawa: Minister of Justice. Disponível em: <http:/ /laws-lois.justice.gc.ca/PDF/T-3.4.pdf>. Acesso em: 30 set. 2016.

CHILE. Decreto 368, de 15 de dezembro de 2010. Disponível em: <http://www.leychile.cl/ Navegar?idNorma=1023845>. Acesso em: 30 set. 2016.

CHILE. Lei 18.168, de 02 de outubro de 1982. Disponível em: <https://www.leychile.cl/ Navegar?idNorma=29591>. Acesso em: 30 set. 2016.

CHILE. Lei 20.453, de 26 de agosto de 2010. Disponível em: <https://www.leychile.cl/ Navegar?idNorma=1016570>. Acesso em: 30 set. 2016.

CRTC. Telecom Decision CRTC 2008-108: The Canadian Association of Internet Providers' Application Regarding Bell Canada's Traffic Shaping of Its Wholesale Gateway Access Service. Ottawa, 2008.

CRTC. Telecom Regulatory Policy CRTC 2009657: Review of the Internet traffic management practices of Internet service providers. Ottawa, 2009.
CRTC. Broadcasting and Telecom Decision CRTC 2015-26: Complaint against Bell Mobility Inc. and Quebecor Media Inc., Videotron Ltd. And Videotron G.P. alleging undue and unreasonable preference and disadvantage in regard to the billing practices for their mobile TV services Bell Mobile TV and illico.tv. Ottawa, 2015.

CRTC. Telecom Notice of Consultation CRTC 2016-192: Examination of differential pricing practices related to Internet data plans. Ottawa, 2016.

CRTC. Telecom Regulatory Policy CRTC 2017104: Framework for assessing the differential pricing practices of Internet service providers. Ottawa, 2017.

DOVAL, Pankaj. Net neutrality: Zero-rating plans must be open to all users says, DoT panel member. The Economic Times. Nova Deli, jul. 2015. Disponível em: <http:// economictimes.indiatimes.com/industry/ telecom/net-neutrality-zero-rating-plans-mustbe-open-to-all-users-says-dot-panel-member / articleshow/48141628.cms>. Acesso em: 20 mar. 2017

EISENACH, Jeffrey A. The economics of zero rating. NERA Economic Consulting, March, 2015 .

FOUAD, Youssef. Regulation clarifying the provision on net neutrality. Iris, 2015. Disponível em: <http://merlin.obs.coe.int/iris/2015/6/ article29.en.html>. Acesso em: 9 mar. 2017.

GARCIA E SILVA, Hermann Bergmann. Neutralidade de rede: a prática do zero-rating e o Marco Civil da Internet. Dissertação (Mestrado em Sistemas de Informação e Gestão do Conhecimento). Belo Horizonte: Universidade FUMEC, 2017. 
GEIST, Michael. Network neutrality in Canada. For sale to the highest bidder: Telecom policy in Canada, p. 73-82, 2008.

HART, Jeffrey A. The teletel/minitel system in France. Pergamon Press, v. 5, n. 1, 1988.

HOLANDA. Telecommunications Act. 2012. Disponível em: <https://www.government.nl/ documents / policy-notes / 2012/06/07/dutchtelecommunications-act>. Acesso em: 7 mar. 2017.

ÍNDIA. Net Neutrality DoT Committee Report. Nova Deli, 2015. Disponível em <http://www. dot.gov.in/sites/default/files/Net_Neutrality_ Committee_report $\% 20 \% 281 \% 29 \_0 . p d f>$ Acesso em: 20 mar. 2017.

LEE, Robin S.; WU, Tim. Subsidizing creativity through network design: zero-pricing and net neutrality. The Journal of Economic Perspectives, v. 23, n. 3, p. 61-76, 2009.

MARQUES, Rodrigo Moreno; KERR PINHEIRO, Marta Macedo. Informação e poder na arena da Internet. Informação \& Sociedade. João Pessoa, v. 24, n. 1, p. 47-60, jan./abr. 2014.

MARSDEN, Christopher T. Comparative case studies in implementing Net Neutrality: a critical analysis of zero rating. SCRIPTed, v. 13, p. 1, 2016.

PRAKASH, Pranesh. Regulatory Perspectives on Net Neutrality. 2015. Disponível em: <http:/ / cisindia.org/internet-governance/blog/regulatoryperspectives-on-net-neutrality>. Acesso em: 20 fev. 2017.

RAMOS, Pedro Henrique Soares. Towards a developmental framework for net neutrality: The rise of sponsored data plans in developing countries. In: 2014 TPRC. Conference Paper. São Paulo Law School of Fundação Getúlio Vargas, 2014.

RAMOS, Pedro Henrique Soares. Arquitetura da rede e regulação: a neutralidade da rede no Brasil. 2015. 218 f. Dissertação (Mestrado em Direito da Escola de Direito da Fundação Getúlio Vargas) - FGV, São Paulo, 2015.

SILVA, Alberto Cerda. Neutralidad de la red y libertad de expresión. Cuestión de Derechos, v. 4, p. 67-78, 2013.

SUBTEL. Ofício Circular no ${ }^{\circ}$ 40/DAP 13221/F-51, de 14 de abril de 2014. Disponível em:

<http://www.subtel.gob.cl/transparencia/ Perfiles/Transparencia20285/Normativas/ Oficios/14oc_0040.pdf>. Acesso em: 30 set. 2016.

TRAI. Prohibition of Discriminatory Tariffs for Data Services Regulations. New Delhi, 2016. Disponível em:

<http://www.trai.gov.in/sites/default/files/ Regulation_Data_Service.pdf $>$. Acesso em: 20 mar. 2017.

TRAI. Recommendations on Net Neutrality. New Delhi, 2017. Disponível em: <http:// www.trai.gov.in/sites/default/files / Recommendations_NN_2017_11_28.pdf>. Acesso em: 15 de. 2017.

UNIÃO EUROPEIA. Regulation 2015/2120. Jornal Oficial, L310/1, 26 de novembro de 2015. Disponível em: <http://eur-lex.europa.eu/eli/ reg/2015/2120/oj>. Acesso em: 8 jul. 2016. 
VAN EIJK, Nico. Net Neutrality in the Netherlands. Journal of Law and Economic Regulation, v. 7, n. 1, p. 146-162, 2014.

VAN SCHEWICK, Barbara. T-Mobile's Binge On Violates Key Net Neutrality Principles. Report. Stanford, CA: Stanford Law School's Center for Internet and Society, 2016.
WU, Tim. Network neutrality, broadband discrimination. Journal on Telecommunications and High Technology Law. v. 2, p. 141, 2003. 